\title{
Cabinets médicaux et hôpitaux, des valeurs du point différentes. Pourquoi?
}

\author{
Jacques-Henri Weidmann, service tarifaire de la FMH
}

Bien que la structure tarifaire TARMED soit unique, de nombreuses personnes se posent la question de savoir pour quelle raison la valeur du point est différente entre la pratique ambulatoire privée (cabinet médical) et le domaine ambulatoire hospitalier. L'explication est à rechercher sur deux plans.

\section{LAMal}

Les valeurs du point sont fixées sur une base cantonale ou régionale. Il existe en principe au moins deux communautés tarifaires ou budgets par canton. L'une de ces communautés tarifaires lie les médecins par le biais de la Société cantonale de médecine à santésuisse. L'autre lie $\mathrm{H}+$ à santésuisse, également sur une base cantonale ou régionale. Dans ce dernier cas, une différentiation est également faite entre hôpitaux publics et privés.

La fixation de la valeur du point à l'introduction du TARMED s'est faite sur la base d'un transcodage du tarif cantonal vers le TARMED. De même, ce transcodage a été fait pour le tarif hospitalier ambulatoire. Un nombre total de points facturés durant une année a ainsi été évalué. Le concept de neutralité des coûts imposant un volume annuel de facturation, celui-ci a été divisé par le nombre total de points facturés durant une année. Le résultat est une valeur du point qui est, par la force des choses, différente entre la pratique privée et la pratique hospitalière ambulatoire.

Le seul canton faisant exception est Genève où une Communauté tarifaire lie les médecins, $\mathrm{H}+$ et santésuisse.

A cela s'ajoute le fait que les médecins ainsi que santésuisse disposent de données permettant une véritable gestion de la neutralité des coûts par le Bureau pour la neutralité des coûts. Il en résulte des adaptations régulières de la valeur du point pour les médecins installés en cabinet. A l'inverse, les données disponibles ne permettent pour l'heure aucune gestion de la valeur du point dans le budget $\mathrm{H}+$ /santésuisse.

Au-delà des notions d'efficacité, d'adéquation et d'économicité ancrées dans la loi, ces différences de valeur du point dans un même Canton interpellent tout citoyen se demandant pour quelle raison une même prestation peut conduire à deux facturations très différentes en fonction du statut du fournisseur de la dite prestation. Il est également étonnant de constater que les hôpitaux publics, subventionnés par l'Etat, bénéficient en règle générale d'une valeur du point supérieure à celle valable pour le médecin installé qui lui ne bénéficie d'aucune subvention.

\section{LAA/LAI/LAM}

Ce sont deux conventions qui lient ici les fournisseurs de prestations et les assureurs. L'une engage les médecins (FMH), l'autre les hôpitaux $(\mathrm{H}+)$. A la date d'introduction du tarif, soit le $1^{\text {er }}$ mai 2003 pour les médecins installés et le $1^{\mathrm{er}}$ janvier 2004 pour les hôpitaux, la valeur du point était fixée à Fr. 1.- dans les deux cas. La période d'évaluation des coûts par cas de 18 mois a débuté le jour de l'introduction du tarif et c'est à la fin de cette période que la valeur du point a été fixée à Fr. - .92 pour les médecins installés. Pour ce qui concerne les hôpitaux, cette phase d'évaluation courre encore jusqu'au 30 juin 2005.

Il n'en demeure pas moins qu'il existe à l'heure actuelle deux valeurs du point différentes à l'hôpital et en pratique privée. Là aussi, c'est le médecin qui doit facturer avec la valeur la plus faible. Il est toutefois possible que cette situation ne soit que provisoire jusqu'au 30 juin de l'année prochaine.

\section{Conclusion}

Les conventions-cadre TARMED (LAMal et LAA/LAI/LAM) définissent des communautés tarifaires séparées pour les médecins et pour les hôpitaux. Les valeurs du point qui en résultent sont différentes pour chacun des fournisseurs de prestation. C'est pour cette raison qu'une convergence de valeur de point ne devrait pas uniquement se faire entre les cantons, mais également entre les divers prestataires de service afin d'harmoniser les tarifs et éviter ainsi des incitations inutiles. 